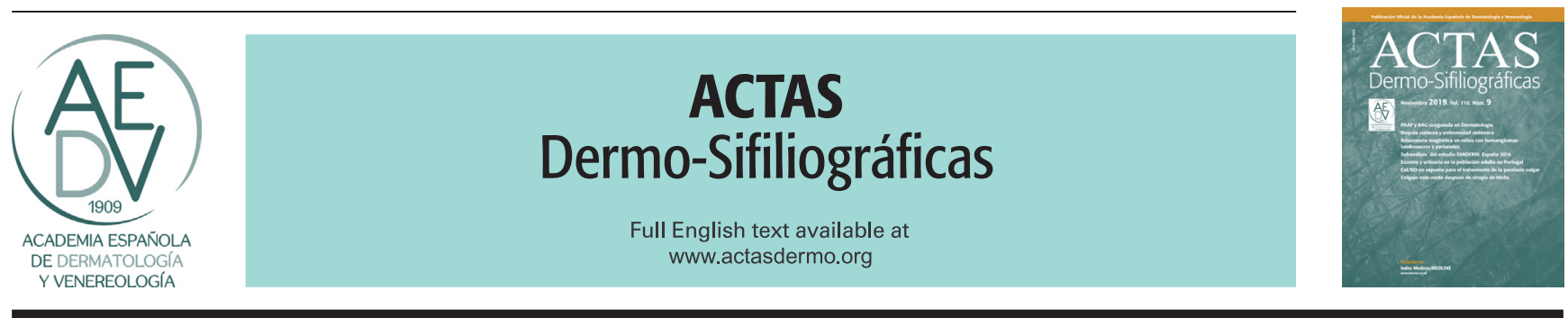

\title{
ORIGINAL
}

\section{Enfermedad de Paget extramamaria}

\author{
J. Marcoval ${ }^{\mathrm{a}, *}$, R.M. Penín ${ }^{\mathrm{b}}$, A. Vidal $^{\mathrm{b}}$ y J. Bermejo ${ }^{\mathrm{c}}$ \\ a Servicio de Dermatología, Hospital Universitari de Bellvitge, Barcelona, España \\ b Servicio de Anatomía Patológica, Hospital Universitari de Bellvitge, Barcelona, España \\ c Servicio de Cirugía Plástica, Hospital Universitari de Bellvitge, Barcelona, España
}

Recibido el 30 de mayo de 2019; aceptado el 15 de septiembre de 2019

Disponible en Internet el 9 de abril de 2020

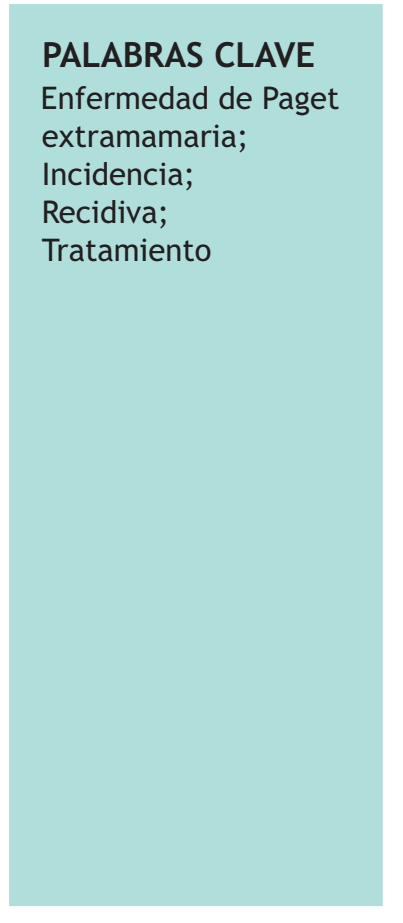

\begin{abstract}
Resumen
Antecedentes y objetivos: Existen pocos estudios sobre la enfermedad de Paget extramamaria (EPEM) en la población mediterránea. Nuestro objetivo fue revisar las características de nuestros pacientes con EPEM, su asociación con neoplasia en continuidad y su evolución a largo plazo.

Pacientes y métodos: Realizamos un estudio observacional retrospectivo sobre 27 pacientes diagnosticados de EPEM entre 1990-2015. Las historias clínicas fueron revisadas retrospectivamente para obtener los datos clínico-patológicos y de seguimiento.

Resultados: Se trata de 20 mujeres y 7 varones de entre 42 y 88 años de edad (mediana de 76 años). Las lesiones se localizaron en la vulva (16 casos), en el pubis-región inguinal (5), en la región perianal (4) y en la axila (2). El tiempo de evolución al diagnóstico osciló entre 1 y 60 meses (mediana de 12 meses) y el diámetro máximo entre 20 y $140 \mathrm{~mm}$ (mediana de $55 \mathrm{~mm}$ ). En 3 casos $(11,1 \%)$ la EPEM fue secundaria. Ningún caso se desarrolló sobre adenocarcinoma anexial cutáneo previo. Diez de 24 EPEM primarias $(41,7 \%)$ presentaban invasión de la dermis. Ocho de los 27 pacientes $(29,6 \%)$ presentaron recidiva local tras el tratamiento quirúrgico inicial. Tres pacientes $(11,1 \%)$ fallecieron a consecuencia de metástasis de la EPEM.

Conclusiones: La presencia de un adenocarcinoma anexial cutáneo subyacente es poco frecuente pero no es rara la existencia de un adenocarcinoma extracutáneo en continuidad. A pesar de que la EPEM suele evolucionar lentamente, es frecuente la invasión de la dermis y no son excepcionales las metástasis. Las recidivas locales son frecuentes a pesar de la extirpación con márgenes amplios y pueden ser tardías, por lo que es preciso un seguimiento a largo plazo. (c) 2020 Publicado por Elsevier España, S.L.U. en nombre de AEDV. Este es un artículo Open Access bajo la licencia CC BY-NC-ND (http://creativecommons.org/licenses/by-nc-nd/4.0/).
\end{abstract}

\footnotetext{
* Autor para correspondencia.

Correo electrónico: jmarcoval@bellvitgehospital.cat (J. Marcoval).
} 


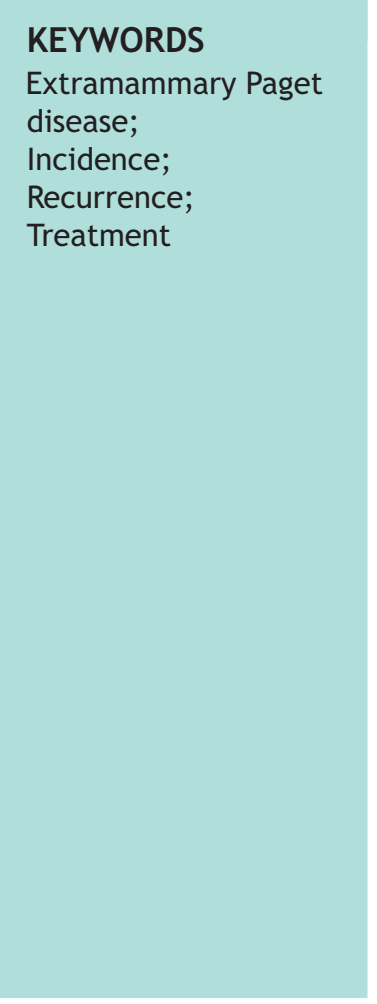

\section{Extramammary Paget Disease}

\section{Abstract}

Background and objective: Extramammary Paget disease (EMPD) has seldom been studied in Mediterranean populations. We aimed to review the characteristics of our patients with EMPD, the presence of a neoplasm in continuity, and the long-term course of the disease.

Patients and methods: Retrospective observational study of 27 patients diagnosed with EMPD between 1990 and 2015. All clinical and pathology findings related to clinical course and outcomes were retrieved for analysis.

Results: Twenty patients were women and 7 were men. Ages ranged from 42 to 88 years (median, 76 years). Lesions were in the following locations: vulva (16 cases), pubis-groin (5), perianal region (4), and axilla (2). Time from onset to diagnosis ranged from 1 to 60 months (median, 12 months) and maximum lesion diameter from 20 to $140 \mathrm{~mm}$ (median, $55 \mathrm{~mm})$. In 3 cases (11.1\%) EMPD was a secondary condition. None of the lesions developed on a previous cutaneous adnexal adenocarcinoma. Ten of the 24 primary EMPDs $(41.7 \%)$ invaded the dermis. Eight of the 27 patients $(29.6 \%)$ experienced local recurrence after the initial surgical treatment. Three patients $(11.1 \%)$ died as a consequence of metastasis from the EMPD.

Conclusions: The presence of an underlying cutaneous adnexal adenocarcinoma is uncommon, but it is not unusual to find an extracutaneous adenocarcinoma in continuity. Although EMPD is a slow-growing tumor, dermal invasion is frequent and metastasis is not uncommon. Local recurrence is common even after excision with wide margins and may be delated, so long term follow-up is essential.

(c) 2020 Published by Elsevier España, S.L.U. on behalf of AEDV. This is an open access article under the CC BY-NC-ND license (http://creativecommons.org/licenses/by-nc-nd/4.0/).

\section{Introducción}

La enfermedad de Paget extramamaria (EPEM) es una entidad poco frecuente resultante de la proliferación intraepidérmica de células malignas de origen glandular fuera de la areola mamaria ${ }^{1,2}$. A pesar de su baja incidencia, recientemente se han publicado extensos estudios sobre la EPEM tanto en Europa como en Asia y en Estados Unidos de América $^{3-8}$. Sin embargo, la mayoría corresponden a estudios epidemiológicos y existen importantes diferencias entre ellos en cuanto a las características clínicas de los pacientes y en la posible asociación a neoplasias subyacentes, algunas de las cuales pueden ser debidas a diferencias raciales y otras pueden deberse al diseño del estudio. Debido a estas discrepancias y a que existen pocos estudios en el Sur de Europa sobre la EPEM nos propusimos revisar nuestros pacientes con EPEM para analizar sus características clínicas, su asociación a neoplasia subyacente en continuidad y su evolución a largo plazo.

\section{Pacientes y métodos}

Realizamos un estudio observacional retrospectivo sobre una serie de 27 pacientes diagnosticados de EPEM entre 1990 y 2015 . Para ello se recogieron los casos registrados como EPEM en la base de datos del Servicio de Anatomía Patológica. Los criterios diagnósticos fueron la presencia de una proliferación intraepidérmica de células atípicas predominantemente aisladas o en pequeños grupos que dispuestas en los estratos parabasal y espinoso adquieren un patrón pagetoide. Todos los casos presentaban expresión inmunohistoquímica de queratinas, sobre todo CK7, EMA y GCDFP-15. La negatividad de S100, MelanA y HMB45 descartó en todos los casos un origen melanocitario. Los casos con adenocarcinoma subyacente fueron diagnosticados de EPEM secundaria. Los pacientes fueron seguidos clínicamente por los Servicios de Dermatología, Ginecología y Cirugía Plástica. Las historias clínicas de los pacientes fueron revisadas retrospectivamente para obtener los siguientes datos: la raza, el sexo y la edad de los pacientes en el momento del diagnóstico, la fecha del diagnóstico, el tiempo de evolución de las lesiones, el diagnóstico clínico de sospecha previo a la biopsia, la ubicación de las lesiones, el diámetro de las lesiones, el tratamiento realizado, el número de recidivas locales, la presencia de tumor infiltrante, la asociación a carcinoma anexial subyacente y/o carcinoma extracutáneo en continuidad, el desarrollo de metástasis, la fecha de la última visita de seguimiento, la fecha del éxitus y si el éxitus fue debido a la EPEM.

Los datos fueron analizados con el paquete estadístico SPSS 17.0 para Windows. Las variables categóricas se compararon mediante la prueba exacta de Fisher. Las variables continuas se compararon mediante la prueba t de Student cuando se confirmó la normalidad de la distribución de datos. De lo contrario, se realizó la prueba $U$ de MannWhitney. La significación estadística se estableció para un valor de $p<0,05$. Se realizaron comparaciones de las parámetros evaluados según el sexo de los pacientes, la presencia de invasión de la dermis subyacente y el desarrollo de recidiva local. También se compararon los parámetros de los casos de EPEM primaria frente a los casos de EPEM secundaria y de los casos que fallecieron a causa de la EPEM frente a los que no fallecieron por EPEM. 

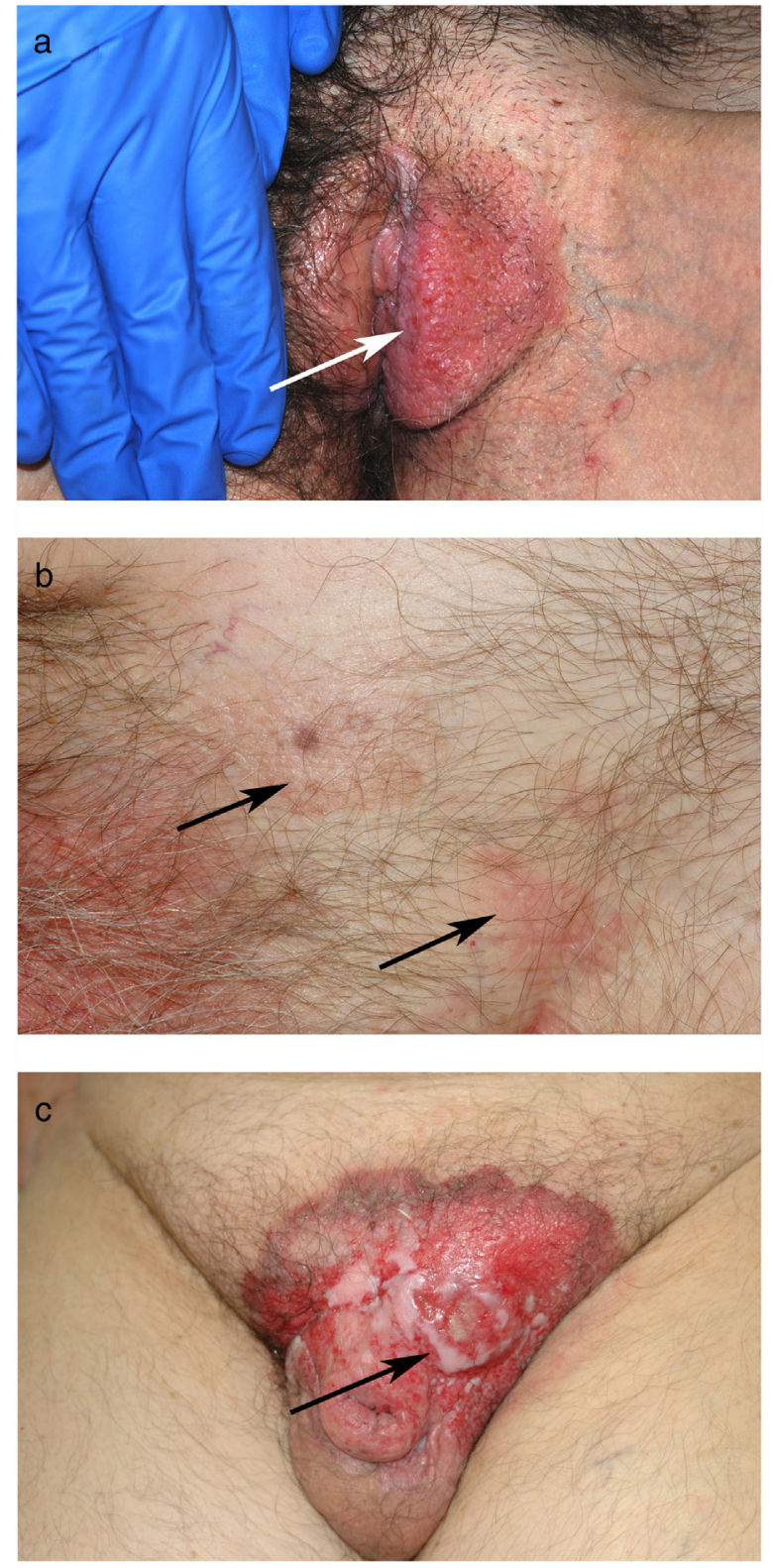

Figura 1 a) Aspecto clínico de la EPEM en el labio mayor izquierdo de la vulva. b) EPEM en el pubis y la región inguinal con 2 áreas aparentemente separadas por piel sana. c) EPEM en el pubis de larga evolución sobre la que se ha desarrollado una lesión tumoral.

\section{Resultados}

Se trata de 20 mujeres y 7 varones, todos ellos de raza blanca. La mediana de edad de los pacientes en el momento del diagnóstico fue de 76 años (intervalo de 42 a 86 años). La mayoría de los pacientes presentaban placas no infiltradas, discretamente eritematosas, algunas de ellas con márgenes bien definidos pero otras mal delimitadas (figs. 1 y 2). Disponemos de información sobre el diagnóstico clínico previo a la biopsia en 21 casos con 26 diagnósticos clínicos propuestos, siendo los más frecuentes el liquen simple (5 casos), el eczema (3 casos), la leucoplasia-eritroplasia (3 casos) y el carcinoma escamoso (2 casos). Otros diagnósticos planteados en una única ocasión fueron la enfermedad de Bowen, el carcinoma basocelular superficial, el intertrigo, los condilomas, la papulosis bowenoide, las dermatofitosis, la psoriasis, la atrofia y la úlcera. Solamente en 4 de 21 casos se sospechó el diagnóstico de la EPEM antes de realizar la biopsia. El tiempo de evolución de las lesiones en el momento del diagnóstico y su localización están detallados en la tabla 1. El diámetro máximo de las lesiones osciló entre 20 y $140 \mathrm{~mm}$ (mediana de $55 \mathrm{~mm}$ ). En 3 casos la EPEM fue secundaria (adenocarcinoma rectal en 2 casos y neoplasia de mama en la axila izquierda en un caso). Ninguno de nuestros pacientes desarrolló la EPEM sobre un adenocarcinoma apocrino previo. Diez de 24 pacientes con EPEM primaria presentaban invasión de la dermis $(41,7 \%)$. En 9 casos la profundidad de invasión fue inferior a $1 \mathrm{~mm}$. El caso restante corresponde a un paciente que rehusó inicialmente el tratamiento y finalmente desarrolló un tumor de $6 \mathrm{~cm}$ de grosor (fig. 1). Se practicó extirpación quirúrgica en 25 pacientes ( 22 de los casos con EPEM primaria y los 3 con EPEM secundaria). Un paciente rehusó el tratamiento quirúrgico y prefirió tratamiento con láser de $\mathrm{CO} 2$ y otro falleció por otros motivos antes del tratamiento.

Ocho de los 27 pacientes $(29,6 \%)$ presentaron al menos una recidiva local tras el tratamiento quirúrgico inicial. Los 8 casos que recidivaron corresponden a EPEM primaria y en 3 de ellos la extirpación quirúrgica inicial presentaba los márgenes libres. Un paciente presentó 6 recidivas locales, otro paciente 5 , dos pacientes presentaron 3 y otros 4 pacientes presentaron una. En los 8 pacientes la primera recidiva se produjo antes de los 3 años del tratamiento quirúrgico inicial pero un paciente presentó sucesivas recidivas a lo largo de 13 años. El tiempo de seguimiento osciló entre 1 y 276 meses (mediana de 60 meses). Tres de nuestros pacientes $(11,1 \%)$ desarrollaron metástasis a distancia y fallecieron debido a metástasis de su EPEM.

La tabla 1 muestra las características clínicas de los pacientes según el sexo. Los casos de EPEM secundaria presentaron una mediana de edad inferior en el momento del diagnóstico (69 frente a 77 años) y una menor mediana del diámetro que los casos primarios ( $30 \mathrm{~mm}$ frente a $57,5 \mathrm{~mm}$ ). Los pacientes que desarrollaron recidiva local presentaron lesiones de mayor diámetro que los que no recidivaron localmente (mediana de $60 \mathrm{~mm}$ frente a $45 \mathrm{~mm}$ ) y una menor mediana de edad en el momento del diagnóstico (65 frente a 77,5 años). Los pacientes que fallecieron debido a la EPEM presentaban lesiones de mayor diámetro que los que no fallecieron (mediana de $80 \mathrm{~mm}$ frente a $52,5 \mathrm{~mm}$ ). Sin embargo, todas estas diferencias no fueron estadísticamente significativas. Tampoco hemos detectado diferencias significativas entre casos con EPEM invasiva y no invasiva en cuanto a la edad de los pacientes, el diámetro de las lesiones ni el retraso diagnóstico. Sin embargo, la presencia de invasión de la dermis sí se asoció con el riesgo de desarrollar metástasis y fallecer a causa de la EPEM (los 3 pacientes que fallecieron corresponden a casos invasivos, $p=0,041)$.

\section{Discusión}

La EPEM y la enfermedad de Paget mamaria se consideran adenocarcinomas intraepidérmicos ${ }^{1}$. La enfermedad de 
Tabla 1 Características clínicas de los pacientes con EPEM según el sexo

\begin{tabular}{|c|c|c|c|}
\hline & Mujer $20(74,1 \%)$ & Varón 7 (25,9\%) & \\
\hline $\begin{array}{l}\text { Edad al diagnóstico } \\
\text { (mediana e intervalo, años) } 76 \text { (42-86) } \\
\text { Localización }\end{array}$ & $73,5(42-86)$ & $79(55-82)$ & $P=0,183$ * \\
\hline Vulva $16(59,3 \%)$ & $16 / 20(80 \%)$ & $0 / 7(0 \%)$ & \\
\hline Pubis-ingle 5 (18,5\%) & $0 / 20(0 \%)$ & $5 / 7(71,4 \%)$ & \\
\hline Perianal $4(14,8 \%)$ & $2 / 20(10 \%)$ & $2 / 7(28,6 \%)$ & \\
\hline Axila $2(7,4 \%)$ & $2 / 20(10 \%)$ & $0 / 7(0 \%)$ & \\
\hline $\begin{array}{l}\text { Diámetro } \\
\text { (mediana e intervalo, mm) } 55(20-140) \\
\text { Retraso diagnóstico }\end{array}$ & $57,5(20-140)$ & $37(25-105)$ & $\mathrm{P}=0,543^{* *}$ \\
\hline (mediana e intervalo, meses) $12(1-60)$ & $12(3-36)$ & $6(1-60)$ & $P=0,326^{*}$ \\
\hline EPEM secundaria $3 / 27(11,1 \%)$ & $1 / 20(5 \%)$ & $2 / 7(28,6 \%)$ & $P=0,156$ \\
\hline EPEM primaria invasiva $10 / 24(41,7 \%)$ & $7 / 19(36,8 \%)$ & $3 / 5(60 \%)$ & $P=0,615$ \\
\hline Tratamiento & & & \\
\hline Cirugía 25 (92,6\%) & $18 / 20(90 \%)$ & $7 / 7$ (100\%) & \\
\hline Radioterapia $3(11,1 \%)$ & $2 / 20(10 \%)$ & & \\
\hline Imiquimod 7 (25,9\%) & $4 / 20(20 \%)$ & $3 / 7$ (50\%) & \\
\hline Láser CO2 1 (3,7\%) & $1 / 20(5 \%)$ & $0 / 7(0 \%)$ & \\
\hline Recidiva local 8/27 $(29,6 \%)$ & $7 / 20(35 \%)$ & $1 / 7(14,3 \%)$ & $P=0,628$ \\
\hline Metástasis $3 / 27(11,1 \%)$ & $2 / 20(10 \%)$ & $1 / 7(14,3 \%)$ & $P=1,000$ \\
\hline Éxitus por EPEM $3 / 27(11,1 \%)$ & $2 / 20(10 \%)$ & $1 / 7(14,3 \%)$ & $P=1,000$ \\
\hline
\end{tabular}

Paget mamaria es debida a la invasión de la epidermis de la areola mamaria por un carcinoma mamario subyacente, mayoritariamente de tipo ductal, que es detectado histológicamente en la mayoría de los casos. La EPEM es una entidad más heterogénea. Por su similitud histológica con la enfermedad de Paget de la mama y su predilección por desarrollarse en áreas ricas en glándulas sudoríparas apocrinas, históricamente se ha considerado originada en adenocarcinomas apocrinos ${ }^{1}$. Sin embargo, en muchos casos no se detecta un adenocarcinoma apocrino subyacente. Algunos autores argumentan que el bajo índice de detección de la supuesta neoplasia anexial subyacente asociada es debida a que el componente in situ en las glándulas apocrinas puede ser pasado por alto en el estudio histológico ${ }^{1}$. También existen casos de EPEM debidos a la diseminación intraepidércima de un adenocarcinoma visceral en continuidad con la piel, como por ejemplo un adenocarcinoma uterino, urotelial o $\mathrm{anal}^{2}$. Los casos que no presentan un adenocarcinoma subyacente se consideran EPEM primaria y cuando existe un adenocarcinoma subyacente se habla de EPEM secundaria.

La teoría más aceptada actualmente es que la mayoría de los casos de EPEM se desarrollan como una neoplasia intraepitelial y por lo tanto corresponden a EPEM primaria. Estos casos se consideran originados a partir de células apocrinas o ecrinas presentes en la epidermis. También se ha sugerido un posible origen a partir de células pluripotenciales epidérmicas, incluyendo las células claras pagetoides del pezón descritas por Toker que se han identificado también en piel perianal ${ }^{9}$. En cuanto a la EPEM secundaria, como se ha comentado anteriormente, puede ser debida a un adenocarcinoma apocrino subyacente o a la diseminación intraepitelial de un adenocarcinoma visceral en continuidad con la piel. En el primero de los casos existen discrepancias en cuanto a la frecuencia con la que se detecta un adenocarcinoma apocrino subyacente que haya invadido la epidermis secundariamente. Por una parte, en una extensa revisión de la literatura, 46 de 153 pacientes presentaban carcinoma anexial cutáneo concurrente lo cual representa aproximadamente el $30 \%$ de casos publicados ${ }^{10}$. Por otra parte, otros estudios detectan solo raramente una asociación a adenocarcinoma anexial cutáneo (por ejemplo, una serie sobre 38 casos con EPEM del pene solo detecta la presencia de adenocarcinoma anexial cutáneo en 3 pacientes) $)^{11}$. En el presente estudio no hemos observado ningún caso originado sobre adenocarcinoma apocrino y creemos que, al menos en nuestra población, la EPEM no suele originarse a partir de un adenocarcinoma apocrino preexistente. Es mucho más frecuente la situación inversa, que la EPEM originada en la epidermis invada la dermis y se convierta así en un adenocarcinoma invasivo. En el segundo de los casos también hay discrepancias en la frecuencia de asociación a un adenocarcinoma visceral en continuidad. Mientras algunos estudios detectan raramente esta asociación (por ejemplo, 0 de 28 casos con lesiones en pene y escroto) ${ }^{11}$, en un extenso estudio publicado recientemente se detectó un adenocarcinoma extracutáneo en continuidad en 37 de 161 casos (23\%) con mayor frecuencia anal-rectal, genital y urotelial ${ }^{7}$. En el caso de la EPEM perianal la frecuencia parece ser incluso más alta puesto que en algunas revisiones de la literatura aproximadamente el $80 \%$ de casos presentaban un adenocarcinoma visceral subyacente, aunque esta asociación puede estar sobreestimada al tratarse de revisiones de casos publicados $^{12,13}$. En nuestra serie hemos observado 3 casos con EPEM secundaria (2 asociados a adenocarcinoma rectal y una paciente con carcinoma de mama en la axila y EPEM en la piel suprayacente), lo cual representa el 11,1\% 
del total de nuestros pacientes y el $50 \%$ de los casos localizados a nivel perianal.

Mención especial merece la posible asociación con adenocarcinoma de próstata. Los niveles elevados de PSA y la expresión inmunohistoquímica de PSA por algunos casos de EPEM han sugerido un posible origen a partir de adenocarcinoma prostático. Sin embargo, en un reciente estudio se ha observado la elevación de PSA y la expresión inmunohistoquímica de PSA en algunos casos de EPEM sin presencia de adenocarcinoma de próstata asociado ${ }^{14}$, por lo que la posible asociación puede estar sobrevalorada quizás debido a la edad avanzada de los pacientes con EPEM. En el presente estudio no hemos observado ningún caso asociado a adenocarcinoma de próstata.

Otra cuestión polémica en la EPEM es que se ha sugerido que puede estar asociada a cáncer visceral a distancia ${ }^{8,10}$. Sin embargo la posible relación patogénica no es clara. Si bien es cierto que existen metástasis dérmicas de adenocarcinomas viscerales que secundariamente pueden invadir la epidermis dando un patrón pagetoide ${ }^{15}$, no pueden considerarse estrictamente EPEM sino metástasis cutáneas a distancia con componente epidermotropo y en este último caso habitualmente pueden distinguirse de la EPEM porque el componente dérmico es mayor que el epidérmico. En un reciente estudio Schmitt et al. ${ }^{7}$ son incapaces de confirmar la existencia de una asociación real o si se trata de una asociación casual debida a edad y proponen eliminar los cánceres que no están en continuidad con las lesiones de EPEM del paraguas de cánceres asociados con la EPEM.

Existen importantes diferencias raciales en cuanto a la incidencia de la EPEM, el sexo de los pacientes afectados y la localización de las lesiones. La incidencia de la EPEM se ha estimado en 0,9 casos por millón de habitantes y año en países occidentales y en 10 casos por millón de habitantes y año en países asiáticos ${ }^{5,16}$. La incidencia que podemos extrapolar en nuestra población de referencia de un millón de habitantes es de aproximadamente un nuevo caso anual por millón, similar a la de otros estudios realizados en la población occidental. En cuanto al sexo, de forma similar a otros estudios en raza blanca ${ }^{7}$, en nuestra población la EPEM también es más frecuente el sexo femenino (20 de 27 de nuestros pacientes son mujeres, 74,01\%) al contrario que en Asia donde parece ser más frecuente en varones ${ }^{6}$. En cuanto a la localización y de acuerdo con otros estudios en pacientes de raza blanca ${ }^{7}$, el área más frecuentemente involucrada en nuestros pacientes es la vulva en las mujeres y la región púbico-inguinal en los hombres, seguidas en ambos sexos por la región perianal. No hemos observado ningún caso en el pene y solamente uno con afectación escrotal a partir del pubis. En cuanto a la edad de los pacientes hay menos discrepancias entre los estudios publicados $y$, al igual que en la mayoría de ellos, la mediana de la edad de nuestros pacientes en el momento del diagnóstico es superior a los 70 años $^{7}$.

Según la literatura, la EPEM clínicamente suele presentarse como una placa eritematosa de lento crecimiento. El margen suele ser bien delimitado, redondeado y ligeramente sobreelevado. La superficie puede ser descamativa ${ }^{17}$. Cuando afecta a mucosas puede presentarse como leucoqueratosis. El primer síntoma, especialmente en las lesiones de la vulva, es el prurito y hay que plantearse este diagnóstico en casos de prurito vulvar especialmente persistente ${ }^{17,18}$.
Sin embargo, el diagnóstico no suele sospecharse antes de la biopsia. En nuestros pacientes el diagnóstico de la EPEM fue sospechado únicamente en 4 pacientes. Esta baja especificidad de la apariencia clínica de las lesiones explica que el tiempo medio de retraso diagnóstico descrito en la literatura sea de aproximadamente 2 años $^{18,19}$ (mediana de 12 meses en el presente estudio).

Aunque no se origine en un adenocarcinoma subyacente, si la EPEM se deja evolucionar puede invadir la dermis y producir metástasis en los ganglios linfáticos regionales e incluso metástasis a distancia ${ }^{6}$. La EPEM primaria ha sido invasiva en el $15-40 \%$ de casos recogidos en una reciente revisión ${ }^{2}$ y en el presente estudio se detectó invasión dérmica en 10 de 24 casos, lo cual representa el $41,67 \%$, porcentaje similar al de otros estudios realizados en nuestro país ${ }^{20}$. Entre los casos invasivos constituyen signos de mal pronóstico una mayor profundidad de invasión, los niveles séricos elevados de CEA, la presencia de invasión linfovascular y el número de ganglios linfáticos regionales invadidos $^{6,19,21}$. Los casos con una profundidad de invasión inferior a $1 \mathrm{~mm}$ se consideran mínimamente invasivos ${ }^{18}$ mientras que una profundidad superior a $4 \mathrm{~mm}$ está asociada a un mayor riesgo de desarrollar metástasis ${ }^{6}$. El riesgo de metástasis en la EPEM invasiva es del 38\% (114 de 301 casos de una serie presentaron metástasis, el $21 \%$ solo en ganglios regionales y el $17 \%$ además metástasis a distancia) ${ }^{6}$. En nuestro estudio, 3 de nuestros pacientes con EPEM invasiva primaria presentaron metástasis a distancia, lo cual representa el $12,5 \%$ de casos de EPEM primaria y el $30 \%$ de casos de EPEM primaria invasiva y todos ellos fallecieron a consecuencia de EPEM. Como en estudios previos, ninguno de nuestros pacientes con EPEM no invasiva ha desarrollado metástasis ${ }^{2}$.

El tratamiento de elección de la EPEM es la extirpación quirúrgica ${ }^{2}$. Algunos estudios aconsejan extirpación con $1 \mathrm{~cm}$ de margen para lesiones con bordes bien definidos y $2 \mathrm{~cm}$ si la lesión no está bien delimitada ${ }^{2,19,22}$. El mapeo biópsico previo puede ser útil en casos mal delimitados y cuando no puedan alcanzarse márgenes de $2 \mathrm{~cm}^{22}$. Sin embargo, el índice de recurrencia tras la cirugía convencional es bastante elevado (entre el $12 \%$ y el $58 \%$ con un promedio del $33 \%)^{18}$. En nuestro estudio presentaron recidiva local 8 pacientes $(29,6 \%)$, algunos de ellos en múltiples ocasiones a pesar de mapeo biópsico previo en los casos mal delimitados. La cirugía de Mohs permite reducir el índice de recurrencias al $8 \%-16 \%^{23,24}$. Recientemente se ha sugerido que la tinción inmunohistoquímica para citoqueatina 7 durante la cirugía de Mohs puede reducir aún más la posibilidad de recidiva pero requiere experiencia y puede alargar el tiempo operatorio ${ }^{25}$. Otros centros realizan biopsias intraoperatorias en congelado para reducir el tiempo operatorio que supondría realizar cirugía de Mohs ${ }^{11}$. Otro dato a tener en cuenta es que los márgenes libres no aseguran la curación puesto que algunos estudios detectan índices de recurrencia elevados, tanto en pacientes con márgenes afectados como en pacientes con márgenes libres (70\% y $37,5 \%$ respectivamente $)^{26}$. Estos datos sugieren que no siempre está justificado realizar intervenciones quirúrgicas mutilantes para obtener márgenes libres. Los casos complejos deberían ser discutidos en un comité multidisciplinario para valorar cada caso individualmente, teniendo en cuenta la decisión del paciente y su familia y el contexto clínico del paciente. En los pacientes en los que no puede realizarse 


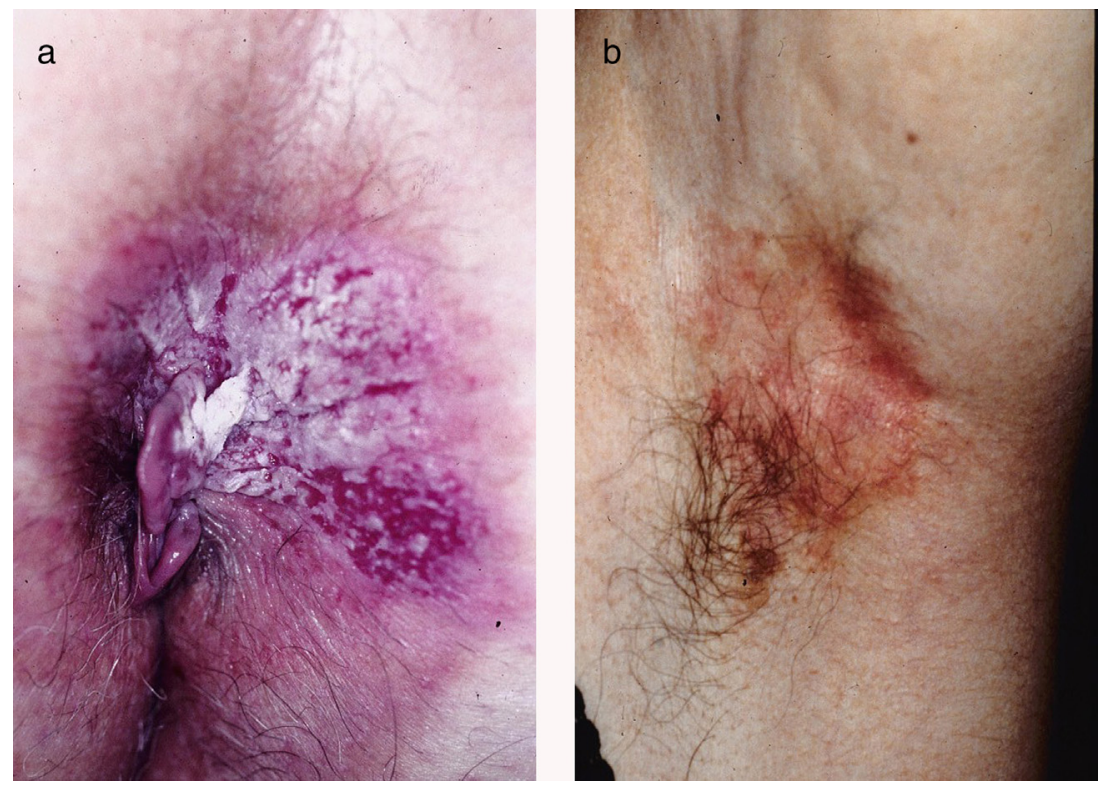

Figura 2 a) Aspecto clínico de EPEM en la región perianal. b) EPEM a nivel axilar.

extirpación quirúrgica se ha utilizado imiquimod tópico con índices de respuesta completa del $56-75 \%$, aunque en más de la mitad de los casos se producen recidivas locales ${ }^{2}$. También puede utilizarse la radioterapia como complemento del tratamiento quirúrgico o como terapia alternativa para pacientes en los que la cirugía está contraindicada o no es factible $^{27}$. La terapia fotodinámica también se ha utilizado con índices de respuesta similares a imiquimod ${ }^{28}$. Puesto que se producen metástasis en los ganglios regionales en el 8,5-26\% de $\operatorname{casos}^{29}$, algunos estudios han evaluado la utilidad de la biopsia del ganglio centinela ${ }^{30,31}$, pero su valor pronóstico es controvertido y su valor terapéutico no está establecido. Si la biopsia del ganglio centinela es positiva o si existe evidencia clínica de afectación ganglionar regional suele realizarse linfadenectomía ${ }^{2}$. Sin embargo, puesto que la EPEM puede ser extensa y suele estar cerca del territorio ganglionar inguinal, la detección del ganglio centinela puede ser técnicamente difícil. Por este motivo en la región vulvar y perianal algunos centros no realizan biopsia del ganglio centinela y practican directamente linfadenectomía en casos de EPEM invasiva con invasión superior a $1 \mathrm{~mm}$ de profundidad, de acuerdo con las directrices del carcinoma vulvar $^{2}$. No existe ningún tratamiento estandarizado para la EPEM diseminada a distancia, pero la mayoría de los trabajos publicados utilizan distintas combinaciones de carboplatino o cisplatino, taxanos y/o 5-fluorouracilo ${ }^{2,32}$.

Para descartar la existencia de posibles neoplasias asociadas algunos autores aconsejan realizar exploraciones complementarias exhaustivas incluyendo test de Papanicolau, mamografía, PSA, colonoscopia, citología de orina, así como el screening apropiado a la edad del paciente ${ }^{7}$. Sin embargo, recientes estudios cuestionan la utilidad de la colonoscopia y aconsejan descartar neoplasias malignas solo cuando la EPEM está en continuidad con ano, uretra o vagina $^{2,33}$. De acuerdo con estos estudios solo estaría indicado realizar citología de orina y test de Papanicolau en los casos de EPEM genital y rectoscopia en los casos de EPEM perianal. En cuanto al seguimiento de los pacientes, debido a que pueden producirse recidivas incluso con márgenes quirúrgicos libres ${ }^{26}$, como ocurrió en 3 de nuestros pacientes, es especialmente importante realizar un seguimiento clínico a largo plazo. Aunque no existen estudios amplios sobre el tema, dado el riesgo de desarrollar metástasis en los casos invasivos puede estar indicado realizar pruebas de imagen en los primeros años de seguimiento.

Las limitaciones del presente estudio son que se trata de un estudio observacional retrospectivo y el limitado número de pacientes.

En resumen, en nuestra población mediterránea la EPEM es más frecuente en mujeres, en la vulva y en personas de más de 70 años. La presencia de adenocarcinoma apocrino subyacente es muy poco frecuente pero sí que es frecuente que la EPEM sea invasiva en el momento del diagnóstico $(41,7 \%)$. Los casos secundarios representan el 11,1\% de pacientes y el $50 \%$ de casos perianales. Las recidivas locales son frecuentes especialmente en lesiones de mayor diámetro y algunos pacientes presentan múltiples recidivas. A pesar de ser una lesión de evolución habitualmente lenta, en nuestro estudio fallecieron 3 pacientes debido a metástasis de EPEM, lo cual representa el $11,1 \%$ del total y el $30 \%$ de los casos primarios invasivos. Puesto que las recidivas locales son frecuentes independientemente del estado de los márgenes de resección y dado que pueden presentarse tras más de 10 años del tratamiento, es preciso realizar un seguimiento a largo plazo de todos los pacientes con EPEM.

\section{Conflicto de intereses}

Los autores declaran no tener ningún conflicto de intereses.

\section{Bibliografía}

1. Lloyd J, Flanagan AM. Mammary and extramammary Paget's disease. J Clin Pathol. 2000;53:742-9. 
2. Ito T, Kaku-Ito Y, Furue M. The diagnosis and management of extramammary Paget's disease. Expert Rev Anticancer Ther. 2018;18:543-53.

3. Siesling S, Elferink MA, van Dijck JA, Pierie JP, Blokx WA. Epidemiology and treatment of extramammary Paget disease in the Netherlands. Eur J Surg Oncol. 2007;33:951-5.

4. Kang Z, Zhang Q, Zhang Q, Li X, Hu T, Xu X, et al. Clinical and pathological characteristics of extramammary Paget's disease: report of 246 Chinese male patients. Int J Clin Exp Pathol. 2015; 8:13233-40.

5. Herrel LA, Weiss AD, Goodman M, Johnson TV, Osunkoya AO, Delman KA, et al. Extramammary Paget's disease in males: survival outcomes in 495 patients. Ann Surg Oncol. 2015;22:1625-30.

6. Ohara K, Fujisawa Y, Yoshino K, Kiyohara Y, Kadono T, Murata $\mathrm{Y}$, et al. A proposal for a TNM staging system for extramammary Paget disease: Retrospective analysis of 301 patients with invasive primary tumors. J Dermatol Sci. 2016;83:234-9.

7. Schmitt AR, Long BJ, Weaver AL, McGree ME, Bakkum-Gamez JN, Brewer JD, et al. Evidence-based screening recommendations for occult cancers in the setting of newly diagnosed extramammary Paget disease. Mayo Clin Proc. 2018;93: 877-83.

8. van der Zwan JM, Siesling S, Blokx WA, Pierie JP, Capocaccia R. Invasive extramammary Paget's disease and the risk for secondary tumours in Europe. Eur J Surg Oncol. 2012;38:214-21.

9. Willman JH, Golitz LE, Fitzpatrick JE. Vulva clear cells of Toker: precursors of extramammary Paget's disease. Am J Dermatopathol. 2005;27:185-8.

10. Chanda JJ. Extramammary Paget's disease: prognosis and relationship to internal malignancy. J Am Acad Dermatol. 1985;13:1009-14.

11. Zhu Y, Ye DW, Chen ZW, Zhang SL, Qin XJ. Frozen section-guided wide local excision in the treatment of penoscrotal extramammary Paget's disease. BJU Int. 2007;100:1282-7.

12. Helvig EB, Graham JH. Anogenital (extramammary) Paget's disease. A clinicopathological study. Cancer. 1963;16:387-403.

13. Grow JR, Kshirsagar V, Tolentino M, Gramling J, Schutte AG. Extramammary perianal Paget's disease: report of a case. Dis Colon Rectum. 1977;20:436-42.

14. Hammer A, Hager $H$, Steiniche T. Prostate-specific antigenpositive extramammary Paget's disease-association with prostate cancer. APMIS. 2008;116:81-8.

15. Petcu EB, Gonzalez-Serva A, Wright RG, Slevin M, Brinzaniuc K. Prostate carcinoma metastatic to the skin as an extramammary Paget's disease. Diagn Pathol. 2012;7:106.

16. Cheng PS, Lu CL, Cheng CL, Lai FJ. Significant male predisposition in extramammary Paget disease: a nationwide population-based study in Taiwan. $\mathrm{Br} \mathrm{J}$ Dermatol. 2014;171:191-3.

17. Calonge E. Tumours of Skin Appendages. En: Rook's Textbook of Dermatology. Ninth edition. Griffiths C, Barker J, Bleiker T, Chalmers R, CreamerD, eds. 2016. London. Wiley. Chapter 138.

18. Shaco-Levy R, Bean SM, Vollmer RT, Jewell E, Jones EL, Valdes $\mathrm{CL}$, et al. Paget disease of the vulva: a study of 56 cases. Eur J Obstet Gynecol Reprod Biol. 2010;149:86-91.
19. Hatta N, Yamada M, Hirano T, Fujimoto A, Morita R. Extramammary Paget's disease: treatment, prognostic factors and outcome in 76 patients. Br J Dermatol. 2008;158:313-8.

20. Blasco-Morente G, Martín-Castro A, Garrido-Colmenero C, Tercedor-Sánchez J. Extramammary Paget disease: a report of 10 cases. Actas Dermosifiliogr. 2015;106:e1-5.

21. Hatta N. Prognostic Factors of Extramammary Paget's Disease. Curr Treat Options Oncol. 2018;19:47.

22. Kaku-Ito Y, Ito T, Tsuji G, Nakahara T, Hagihara A, Furue $M$, et al. Evaluation of mapping biopsies for extramammary Paget disease: A retrospective study. J Am Acad Dermatol. 2018;78:1171-7.

23. O’Connor WJ, Lim KK, Zalla MJ, Gagnot M, Otley CC, Nguyen $\mathrm{TH}$, et al. Comparison of Mohs micrographic surgery and wide excision for extramammary Paget's disease. Dermatol Surg. 2003;29:723-7.

24. Hendi A, Brodland DG, Zitelli JA. Extramammary Paget's disease: surgical treatment with Mohs micrographic surgery. J Am Acad Dermatol. 2004;51:767-73.

25. Damavandy AA, Terushkin V, Zitelli JA, Brodland DG, Miller CJ, Etzkorn JR, et al. Intraoperative Immunostaining for Cytokeratin-7 During Mohs Micrographic Surgery Demonstrates Low Local Recurrence Rates in Extramammary Paget's Disease. Dermatol Surg. 2018;44:354-64.

26. Black D, Tornos C, Soslow RA, Awtrey CS, Barakat RR, Chi DS. The outcomes of patients with positive margins after excision for intraepithelial Paget's disease of the vulva. Gynecol Oncol. 2007;104:547-50.

27. Tagliaferri L, Casà C, Macchia G, Pesce A, Garganese G, Gui $B$, et al. The Role of Radiotherapy in Extramammary Paget Disease: A Systematic Review. Int J Gynecol Cancer. 2018;28: 829-39.

28. Rioli DI, Samimi M, Beneton N, Hainaut E, Martin L, Misery L, et al. Efficacy and tolerance of photodynamic therapy for vulvar Paget's disease: a multicentric retrospective study. Eur J Dermatol. 2018;28:351-5.

29. Kusatake K, Harada Y, Mizumoto K, Kaneko S, Niihara H, Chinuki $Y$, et al. Usefulness of sentinel lymph node biopsy for the detection of metastasis in the early stage of extramammary Paget's disease. Eur J Dermatol. 2015;25:156-61.

30. Fujisawa Y, Yoshino K, Kiyohara Y, Kadono T, Murata Y, Uhara H, et al. The role of sentinel lymph node biopsy in the management of invasive extramammary Paget's disease: Multi-center, retrospective study of 151 patients. J Dermatol Sci. 2015;79: $38-42$.

31. Ogata D, Kiyohara Y, Yoshikawa S, Tsuchida T. Usefulness of sentinel lymph node biopsy for prognostic prediction in extramammary Paget's disease. Eur J Dermatol. 2016;26:254-9.

32. Padrnos L, Karlin N, Halfdanarson TR. Mayo Clinic Cancer Center Experience of Metastatic Extramammary Paget Disease 19982012. Rare Tumors. 2016;8:6804.

33. Hegarty PK, Suh J, Fisher MB, Taylor J, Nguyen TH, Ivan D, et al. Penoscrotal extramammary Paget's disease: the University of Texas M D. Anderson Cancer Center contemporary experience. J Urol. 2011;186:97-102. 